\title{
Calculating Pre-SN Mass-Loss Histories from Post-Explosion Observations and the Special Case of SN 2006gy
}

\author{
Ori D. Fox \\ Department of Astronomy, University of California, Berkeley, CA 94720-3411. \\ email: of ox@berkeley.edu
}

\begin{abstract}
A growing number of SNe now show evidence for significant interaction between the forward shock and a pre-existing circumstellar medium (CSM) formed by the pre-SN mass-loss. The SN community likes to argue that it can indirectly constrain the progenitor systems for these SNe by measuring the wind speeds, densities, compositions, and asymmetries of the CSM. The measurements, however, rely on a number of assumptions and can result in degeneracies that straddle the divide between RSGs and more massive stars. Furthermore, the implications of the measured properties may sometimes be simplified to fit a SN expert's relatively basic understanding of stellar wind parameters. Given the opportunity to have experts in massive stars, cool stars, and supernovae together in the same room, I thought it would be a good opportunity to review the different methods employed by the SN community to measure massloss rates. I will then provide an update from a recent Spitzer survey on the measured CSM properties from different subclasses of SNe with known shock interaction (including SLSNe, SNe II, IIn, Ibc, and even Ia-CSM). Finally, I will present new HST data on the SLSN Type IIn SN 2006 gy and discuss the implications on the progenitor system and pre-SN mass-loss.
\end{abstract}

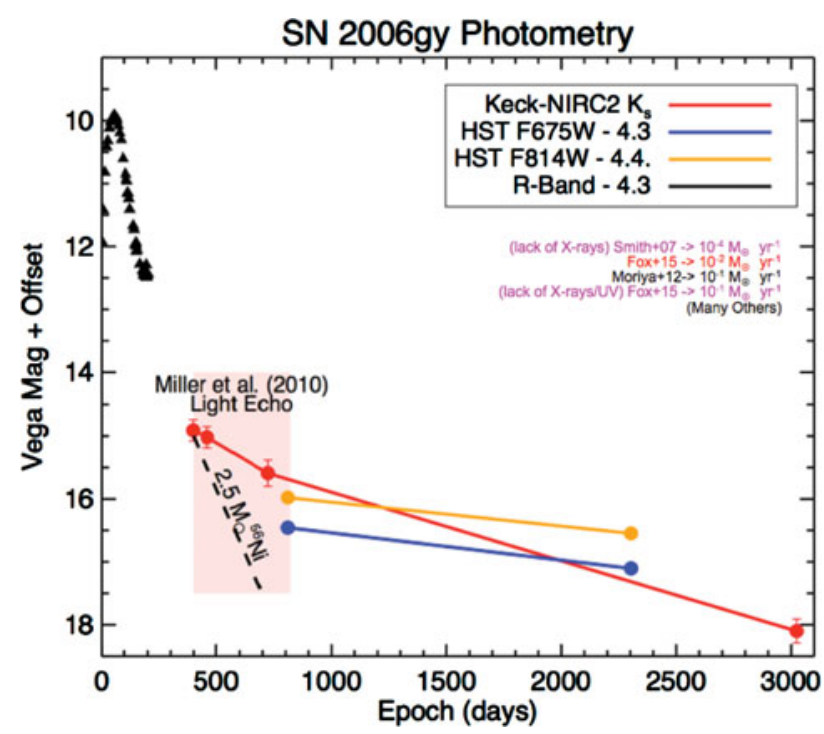

Figure 1. Light curve of SN 2006gy through day 3024, including data from Smith et al. (2008), Miller et al. (2010), and Fox et al. (2015). Offsets are applied only for plot clarity. Similar to other SNe, mass-loss rates have been derived for SN 2006gy using multi-wavelength data. The mass-loss rates and associated references are listed in the figure. The various techniques can often yield wide-ranging results, sometimes up to two orders of magnitude. 


\section{References}

Fox, O. D., Smith, N., Ammons, S. M., et al. 2015, arXiv, 1509.06407

Miller, A. A., Smith, N., Li, W., et al. 2010, AJ, 139, 2218

Smith, N., Foley, R. J., Bloom, J. S., et al. 2008, ApJ, 686, 485 